\title{
Transmit Power and Bit Allocations for OFDM Systems in a Fading Channel
}

\author{
Jiho Jang ${ }^{*}$, Kwang Bok Lee ${ }^{\dagger}$, and Yong-Hwan Lee ${ }^{\dagger}$ \\ ${ }^{*}$ Samsung Electronics Co. Ltd., Suwon P.O.Box 105, Suwon-si, Gyeonggi-do 442-742, Korea \\ ${ }^{\dagger}$ School of Electrical Engineering, Seoul National University, Seoul 151-742, Korea \\ E-mail: jiho@mobile.snu.ac.kr, \{klee, ylee\}@snu.ac.kr
}

\begin{abstract}
In this paper, we propose a computationally efficient algorithm for transmit power and bit allocations in wireless OFDM systems aiming at maximization of data rate under the constraint of total transmit power and bit error rate. Although the proposed algorithm is based on the water-filling approach, the computational complexity is only $O(k \times M)$, where $k$ denotes the number of iterations required to meet the constraints and $M$ is the number of subchannels. By computer simulations, we verify that 10 iterations are sufficient to satisfy the constraints, and the decrease of data rate for the proposed algorithm is shown to be less than one percent relative to the optimal method at the average SNR above $4 \mathrm{~dB}$. The proposed algorithm is appropriate for OFDM systems in a time varying wireless channel for its low computational complexity and good performance.
\end{abstract}

\section{INTRODUCTION}

The growing demand for multimedia services requires reliable and high rate data communications, but the high rate data transmission is significantly limited by intersymbol interference (ISI). Recently, a multicarrier system has been taken a great interest as a solution to the problem of transmitting data over channels with severe ISI [1 5]. The principle of the multicarrier system is to split a high rate data stream into a number of lower rate parallel streams that are transmitted simultaneously on a number of orthogonal subchannels. In the multicarrier system, when the channel state information (CSI) is available at the transmitter, transmit power and number of bits to be transmitted for each subchannel can be adapted according to the CSI in order to reduce transmit power consumption or to increase data rate [6 10].

The algorithm to adaptively allocate transmit power and bits for each subchannel in the multicarrier system is known as a loading algorithm for the application of Asymmetric Digital Subscriber Lines (ADSL), where a discrete multitone (DMT) technology is generally used. Existing loading algorithms in [6], [7], [8], and [9], which are developed for DMT systems, inherently assume that the channel is quasistatic. In the quasi-static channel, the channel state can be assumed not to vary with time. Therefore, the transmitter allocates transmit power and bits once at the beginning, and the allocation may not be changed for a long time. The computational complexity of the loading algorithm in this case may not be a problem, if the transmit power and bit allocations can be done within the time period given for the initial loading.

However, in Orthogonal Frequency Division Multiplexing (OFDM) systems, which are popular in these days for the wireless application of the multicarrier system, the channel state may vary with time. Thus low complexity and efficient computation are important for the loading algorithm applied to the OFDM systems in order to adapt transmit power and number of bits for each subchannel within the coherence time of the wireless channel. Leke and Cioffi developed a loading algorithm for a wireless multicarrier system in [10], but the computational complexity of the algorithm is still high.

In this paper, we propose a computationally efficient loading algorithm for OFDM systems in a time varying wireless channel. We aim at maximization of data rate under the constraint of total transmit power and bit error rate (BER). The proposed loading algorithm is based on the water-filling approach [11], which is known as optimal to maximize data rate under the constraint of total transmit power. In the proposed algorithm, however, the water-filling power allocation is not fully performed. Instead, by adjusting only the water-filling level needed in the water-filling power allocation, we may adapt transmit power and number of bits for each subchannel with low computational complexity, and the total number of loaded bits in an OFDM symbol can be maximized while satisfying the constraints.

This paper is organized as follows. In next section, existing loading algorithms are briefly reviewed. In Section III, we formulate the problem of transmit power and bit allocations for OFDM systems considered in this paper, and propose a loading algorithm appropriate for the wireless OFDM system. Simulation results are shown in Section IV, and we conclude this paper in Section V.

\section{EXISTING ALGORITHMS}

In [6], Hughes-Hartogs developed a loading algorithm to minimize transmit power consumption under the requirements of data rate and BER. This algorithm achieves optimal performance using a greedy search method, where one additional bit is allocated to the subchannel that requires the least incremental power at each step until the target data rate is reached. Since the Hughes-Hartogs' algorithm requires 
exhaustive sorting and adds one more bit at a time, the order of operations is expected to be $O\left(\hat{B}_{H H} \times M \times \log _{2} M\right)$, where $\hat{B}_{H H}$ denotes the total number of loaded bits and $M$ is the number of subchannels.

Krongold et al. treated the data rate maximization problem under the constraint of total transmit power and BER in [7]. They used a table lookup method with precomputed rate-SNR tables, and a bisection method to obtain an optimal Lagrange multiplier solution. The amount of transmit power and number of bits to be allocated for each subchannel are obtained from the value of Lagrange multiplier and the corresponding operating point in the lookup table. This algorithm requires large size of memory to keep all the values of operating points on rate-SNR curve. Also, since the Krongold's algorithm needs table lookups at the initialization stage and at each iteration, and each table lookup requires the operations of $O(M \times D)$, where $D$ denotes the maximum number of bits mapped for a symbol in the constellation, the order of operations for the algorithm is $O((k+1) \times M \times D)$, where $k$ denotes the required number of iterations.

Chow and Cioffi proposed a suboptimal loading algorithm in [8] and [9], where they optimized transmission bandwidth to maximize data rate under the constraint of flat on/off power allocation and BER. In this algorithm, total transmit power is equally distributed over turned-on subchannels, which are allowed to transmit data bits, and then bits are allocated for each subchannel with the strategy that large number of bits for subchannels with high gain, and small number of bits for subchannels with low gain. Also in [10], Leke and Cioffi developed an efficient implementation method of the water-filling power allocation by decoupling the transmission bandwidth identification from the transmit power allocation as in [8] and [9]. The Leke's water-filling power allocation method yields higher data rate than the Chow's flat power allocation scheme.

When the restriction of integer bit constellation is given as in [9] and [10], the number of loaded bits is rounded off to be an integer number, and the transmit power for each subchannel is rescaled accordingly to meet the BER constraint. However, since the rescaled transmit power may not satisfy the total transmit power constraint, the number of loaded bits for each subchannel should be adjusted at each iteration and the procedure should be repeated until the total transmit power constraint can be met. Unfortunately however the iteration procedures are not fully described in [9] and [10] Since the Chow's aglorithm and Leke's algorithm require sorting of the channel power gains at the transmission bandwidth identification stage, the order of operations for both algorithms is expected to be $O\left(M \times \log _{2} M+(k+1) \times M\right)$, where $k$ denotes again the required number of iterations.

\section{PROPOSED ALGORITHM}

In this section, we propose a simple and efficient loading algorithm that maximizes data rate of the OFDM system under the constraints of total transmit power and BER.

\section{A. Problem Formulation}

In OFDM systems, since the total bandwidth is divided into $M$ subchannels and the loading algorithm assigns a certain number of bits, $b_{m}$ for the $m$ th subchannel, the total number of loaded bits in one OFDM symbol is written as

$$
B=\sum_{m=1}^{M} b_{m}
$$

The number of loaded bits, $b_{m}$ for the $m$ th subchannel may be obtained by [9]

$$
b_{m}=\log _{2}\left(1+\frac{s_{m} g_{m}}{\sigma^{2} \Gamma}\right)
$$

where $s_{m}$ and $g_{m}$ denote the transmit power allocated to the $m$ th subchannel, and the power gain of the $m$ th subchannel, respectively. $\sigma^{2}$ is the variance of the additive white Gaussian noise. $\Gamma$ represents the SNR gap which is a function of the target BER and a channel coding scheme. Note that $b_{m}$ in (2) may have a real number in this form, and hence we round off to make $b_{m}$ be an integer number for practical modulation/demodulation as in [9] and [10] such that

$$
\hat{b}_{m}=\operatorname{round}\left[b_{m}\right] \text {, for } m=1,2, \cdots, M \text {. }
$$

Then, the total number of loaded bits in an OFDM symbol can be rewritten as

$$
\hat{B}=\sum_{m=1}^{M} \hat{b}_{m}
$$

Consequently, the problem considered in this paper may be formulated as

$\max \hat{B}$,

$$
\text { subject to } B E R \leq B E R_{\text {target }} \text { and } \sum_{m=1}^{M} \hat{s}_{m} \leq S \text {, }
$$

where $B E R_{\text {target }}$ represents the maximum allowable BER, $\hat{S}_{m}$ denotes the allocated transmit power corresponding to the integer number of bits, $\hat{b}_{m}$ for the $m$ th subchannel, and $S$ is the total available transmit power budget.

\section{B. Algorithm}

Under the assumption that the number of loaded bits can have infinite granularity in constellation size, the optimal transmit power allocation policy that maximizes the total number of loaded bits (1) can be easily derived using a Lagrange multiplier technique. The solution is the wellknown water-filling [11] over the subchannels, and the transmit power allocated to the $m$ th subchannel can be written as

$$
s_{m}=\sigma^{2} \Gamma\left[\lambda-\frac{1}{g_{m}}\right]^{+},
$$

where $[x]^{+}=\max \{x, 0\} \quad$, and $\lambda$ denotes a threshold representing the water-filling level. By replacing $s_{m}$ in (2) with (6), the number of bits $b_{m}$ for the $m$ th subchannel can be reexpressed as 


$$
b_{m}=\left[\log _{2}\left(\lambda g_{m}\right)\right]^{+} .
$$

After the number of bits $b_{m}$ for $m=1,2, \cdots, M$ is calculated from (7) for a specific $\lambda$, integer number of bits is obtained by rounding off it to be $\hat{b}_{m}$ as (3). Then, corresponding to the obtained integer number of bits $\hat{b}_{m}$, the transmit power for each subchannel should be rescaled to satisfy the BER constraint [9][10]. Assuming that QAM modulation and ideal phase detection are used as in [12], the BER for the $m$ th subcarrier signal is bounded by

$$
B E R \leq \frac{1}{5} \exp \left(-1.5 \frac{\gamma_{m}}{2^{\hat{b}_{m}}-1}\right),
$$

where $\gamma_{m}$ denotes the signal to noise ratio (SNR) for the $m$ th subchannel which is defined by $\gamma_{m}=\hat{s}_{m} g_{m} / \sigma^{2}$. Under the requirement of $B E R \leq B E R_{\text {target }}$, the transmit power for the $m$ th subchannel corresponding to $\hat{b}_{m}$ may be calculated as

$$
\hat{s}_{m}=\frac{\sigma^{2} \Gamma}{g_{m}}\left(2^{\hat{b}_{m}}-1\right)
$$

where the SNR gap, $\Gamma$ in this case is written as $\Gamma=\frac{-\ln \left(5 B E R_{\text {target }}\right)}{1.5}$.

Note from (7) and (3) that $b_{m}$ and $\hat{b}_{m}$ are determined by only the value of the water-filling level, $\lambda$, when the channel power gain $g_{m}$ is given. Therefore, we need not perform the whole water-filling power allocation in [10], but only adjust the value of $\lambda$ to satisfy the total transmit power constraint given as $\sum_{m=1}^{M} \hat{s}_{m} \leq S$ in (5). Note that the number of bits $b_{m}$ for each subchannel increases with $\lambda$ as seen from (7). The increase of $b_{m}$ with $\lambda$ indicates the increase of $\hat{b}_{m}$ and $\hat{s}_{m}$ as well from (3) and (9). Therefore, to maximize the data rate of the system, the water-filling level $\lambda$ should be kept as large as possible while satisfying the total transmit power constraint. Consequently, by adjusting $\lambda$ iteratively, we can adapt transmit power and number of bits for each subchannel, and the number of loaded bits in an OFDM symbol can be maximized while satisfying the total transmit power constraint and BER requirement.

At each iteration, the water-filling level $\lambda$ is adjusted as

$$
\lambda \leftarrow \lambda+\mu \frac{1}{M_{O N}} \cdot \frac{1}{\sigma^{2} \Gamma}\left(S-\sum_{m=1}^{M} \hat{s}_{m}\right),
$$

where $0<\mu<1$ is a step size, and $M_{O N}$ denotes the number of turned-on subchannels which are allowed to transmit data bits. For the initial value of $\lambda$, we may set

$$
\lambda=\frac{1}{M}\left(\frac{S}{\sigma^{2} \Gamma}+\sum_{m=1}^{M} \frac{1}{g_{m}}\right),
$$

which can be obtained from (6) and the condition of $\sum_{m=1}^{M} s_{m}=S$ temporarily assuming that all the subchannels are turned on and relaxing the restriction of the integer bit constellation.
Table 1. Complexity comparisons

\begin{tabular}{|c|c|}
\hline Loading Algorithms & Order of Operations \\
\hline \hline Hughes-Hartogs' algorithm [6] & $O\left(\hat{B}_{H H} \times M \times \log _{2} M\right)$ \\
\hline Krongold's algorithm [7] & $O((k+1) \times M \times D)$ \\
\hline Chow's flat power allocation [9] & $O\left(M \times \log _{2} M+(k+1) \times M\right)$ \\
\hline Leke's water-filling method [10] & $O\left(M \times \log _{2} M+(k+1) \times M\right)$ \\
\hline Proposed & $O(k \times M)$ \\
\hline
\end{tabular}

( $\hat{B}_{H H}$ : total number of loaded bits, $M$ : number of subchannels, $k$ : required number of iterations, $D$ : maximum number of bits mapped for a symbol in the constellation)

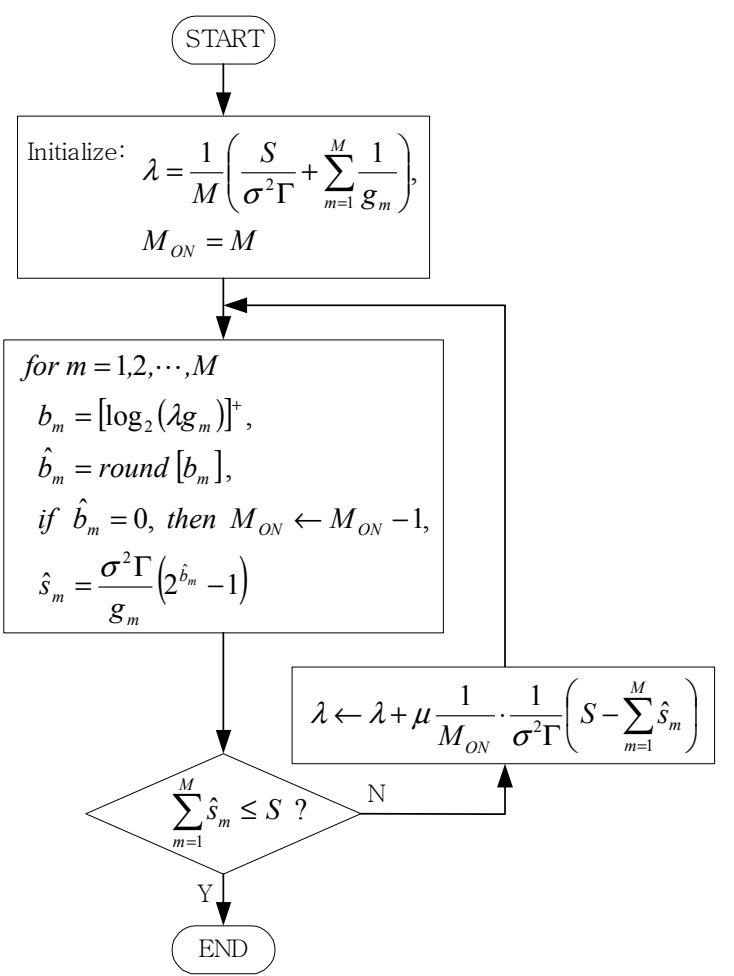

Fig. 1. Flow chart of the proposed loading algorithm

The followings are the summary of the proposed loading algorithm, and the flow chart is depicted in Fig. 1.

(Step 1) Initialize $\lambda$ as (11), set $M_{O N}=M$, and give a specific value for the step size $\mu$.

(Step 2) For $m=1,2, \cdots, M$, calculate $b_{m}, \hat{b}_{m}$, and $\hat{s}_{m}$ from (7), (3) and (9), respectively. If $\hat{b}_{m}=0$ (or $\left.\hat{s}_{m}=0\right)$, then set $M_{O N} \leftarrow M_{O N}-1$.

(Step 3) If $\sum_{m=1}^{M} \hat{s}_{m} \leq S$, then STOP. Allocate the integer 
number of bits $\hat{b}_{m}$, and the transmit power $\hat{s}_{m}$ for each subchannel. Otherwise, adjust $\lambda$ as (10) and GOTO (Step 2).

Note that the proposed algorithm does not require sorting nor table lookup, and thus the algorithm requires the operations of $O(k \times M)$ only. Consequently, the proposed algorithm's computational complexity is low, and the algorithm can be applied to the wireless OFDM systems. The order of operations for the proposed algorithm and those for other algorithms are tabulated in Table 1. The required number of iterations and data rate performance will be shown in next section.

\section{SIMULATION RESULTS}

In this section, we evaluate the performance of the proposed loading algorithm in terms of data rate and required number of iterations by computer simulations. In simulations, the number of subchannels, $M$ is set to be 256 , and we assume that OFDM symbols undergo Rayleigh fading. We consider a wideband multipath fading in this paper, but the bandwidth of each subchannel is small enough to be assumed flat fading for each subcarrier signal. The average channel power gain for each subcarrier signal is assumed to be one, and the average received SNR can be written as $S /\left(M \sigma^{2}\right)$. Also we assume that no channel coding scheme is used, and the target BER is set to be $B E R_{\text {target }}=10^{-3}$. Note that in this case, the SNR gap $\Gamma$ is $5.48 \mathrm{~dB}$.

In figures 2 and 3 , to show the effects of the step size $\mu$ in the proposed algorithm, we depict the Data-Rate-Decrease relative to the optimal method, and the number of iterations required to meet the constraints, respectively, for various values of $\mu$. The optimal method is the modified version of the Hughes-Hartogs' algorithm [6], where a greedy search method is used to maximize data rate under the constraints of total transmit power and BER. The Data-Rate-Decrease relative to the optimal method is defined in this paper by

$$
\text { Data }- \text { Rate }- \text { Decrease }=\frac{\Delta \hat{B}_{H H}-\hat{B}}{\hat{B}_{H H}} \times 100[\%],
$$

where $\hat{B}_{H H}$ represents the total integer number of bits loaded in an OFDM symbol when the Hughes-Hartogs' algorithm is used. In the figures, the results of 10,000 independent trials are averaged to get the Data-Rate-Decrease and the required number of iterations.

Note from Fig. 2 that the Data-Rate-Decrease decreases for all the values of $\mu$ as the average SNR increases. At a fixed average SNR value, however, the Data-Rate-Decrease increases with $\mu$. From Fig. 3, we may observe that the required number of iterations decreases as $\mu$ increases at a fixed average SNR value. The required number of iterations is less than 10 at all the values of average SNR for the cases of $\mu=0.7, \mu=0.8$, and $\mu=0.9$. Considering the tradeoff between the Data-Rate-Decrease and the required number of iterations from the results in Fig. 2 and Fig. 3, the value of

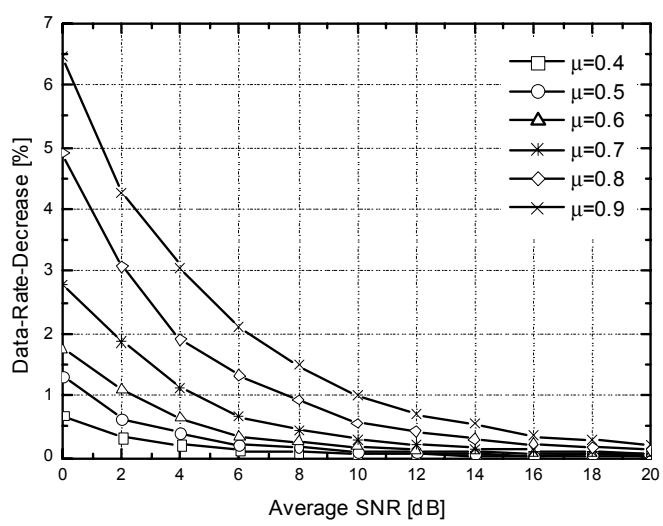

Fig. 2. Data-Rate-Decrease for the proposed algorithm for various values of $\mu$

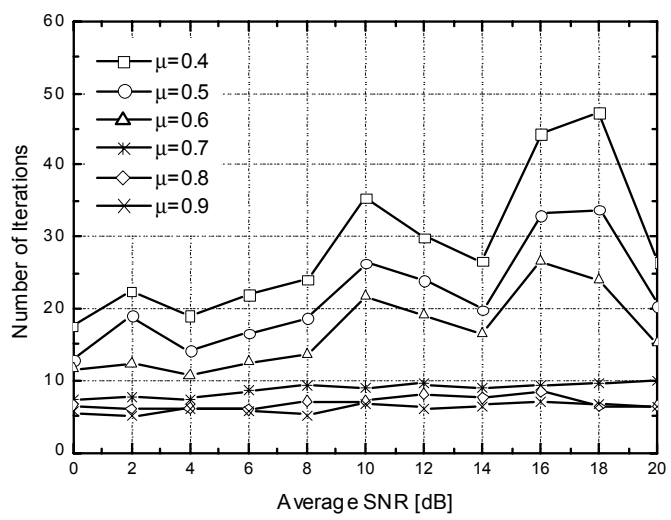

Fig. 3. Required number of iterations for the proposed algorithm for various values of $\mu$

$\mu$ is chosen to be 0.7 for the proposed algorithm, because the Data-Rate-Decrease for the case of $\mu=0.7$ is the least among the values of $\mu$ that require less than 10 iterations.

In figures 4 and 5, we depict the Data-Rate-Decrease, and the required number of iterations, respectively, for various loading algorithms. Note from Fig. 4 that the Data-RateDecrease for the proposed algorithm decreases with average SNR, and the Data-Rate-Decrease is less than one percent relative to the optimal method at average SNR above $4 \mathrm{~dB}$. Moreover, at the average SNR higher than $12 \mathrm{~dB}$, the DataRate-Decrease for the proposed algorithm is less than 0.2 percent and the proposed algorithm shows better performance than all other methods. Also note from Fig. 5 that the proposed algorithm requires less than 10 iterations at all the values of average SNR. The required number of iterations for the Leke's water-filling power allocation method is $4 \sim 9$ according to the average SNR values, and is the least among 
all the methods. However, considering the computational complexity given in Table 1 and the required number of iterations shown in Fig. 5, we may conclude that the proposed algorithm has lower computational complexity than all other methods, because the proposed algorithm requires less than 10 iterations and the order of operations for the proposed algorithm is only $O(k \times M)$.

\section{CONCLUSIONS}

In this paper, we propose a simple and efficient loading algorithm to adaptively allocate transmit power and bits for each subchannel in wireless OFDM systems. We aim at the maximization of data rate under the constraint of total transmit power and BER. The proposed algorithm is based on the well-known water-filling approach. In the proposed algorithm, however, we need not perform the whole waterfilling procedures. Instead, by adjusting only the water-filling level, which is needed in the water-filling power allocation, we adapt transmit power and number of bits for each subchannel with low computational complexity, and data rate is maximized while satisfying the constraints.

The proposed algorithm requires the operations of $O(k \times M)$ only, where $k$ and $M$ represent the required number of iterations and the number of subchannels, respectively. By computer simulations, we verify that 10 iterations are sufficient to meet the constraints for the proposed algorithm. Moreover, simulation results show that the Data-Rate-Decrease for the proposed algorithm is negligible relative to the optimal method, i.e. the Data-RateDecrease is less than one percent at the average SNR above $4 \mathrm{~dB}$. Consequently, the proposed loading algorithm is appropriate for OFDM systems in a time varying wireless channel for its low computational complexity and good performance.

\section{REFERENCES}

[1] J. A. C. Bingham, "Multicarrier modulation for data transmission: an idea whose time has come," IEEE Commun. Mag., pp. 5-14, May 1990.

[2] L. J. Cimini, "Analysis and simulation of a digital mobile channel using orthogonal frequency division multiplexing," IEEE Trans. Commun., vol. COM-33, pp. 665-675, July 1995.

[3] J. Jang, K. B. Lee, and Y. -H. Lee, "Frequency-time domain transmit power adaptation for a multicarrier system in fading channels," IEE Electronics Letters, vol. 38, No. 5, pp. 218-220, 28th Feb. 2002.

[4] J. Jang and K. B. Lee, "Transmit power adaptation for multiuser OFDM systems," IEEE J. Select. Areas Commun., vol. 21, No. 2, pp. 171-178, Feb. 2003.

[5] J. Jang, K. B. Lee, and Y. -H. Lee, "Frequency-time domain transmit power adaptation for OFDM systems in a multiuser environment," IEE Electronics Letters, vol. 38, No. 25, pp. 1754-1756, 5th Dec. 2002.

[6] D. Hughes-Hartogs, "Ensemble modem structure for imperfect transmission media," U.S. Patents Nos. 4,679,227 (July 1987), 4,731,816 (March 1988), and

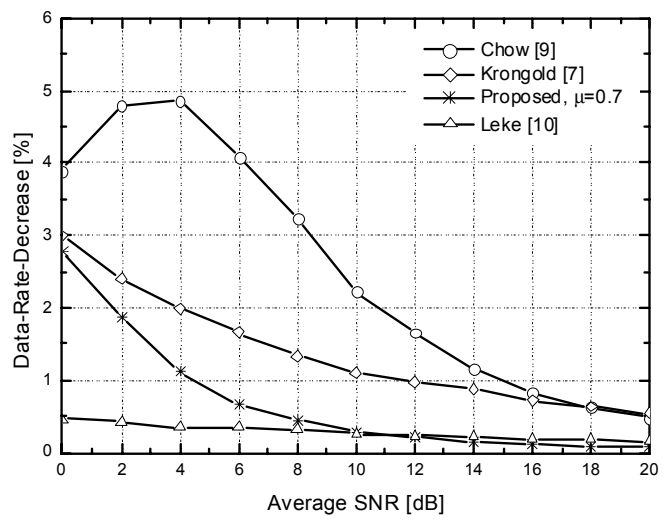

Fig. 4. Data-Rate-Decrease for various loading algorithms

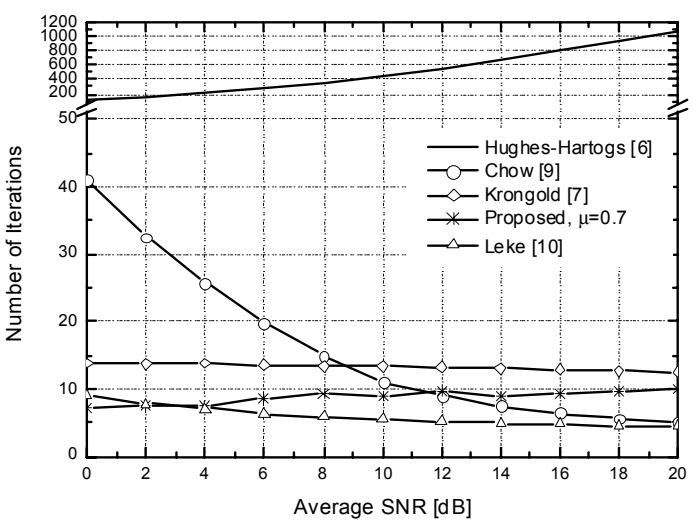

Fig. 5. Required number of iterations for various loading algorithms

4,833,706 (May 1989).

[7] B. S. Krongold, K. Ramchandran, and D. L. Jones, "Computationally efficient optimal power allocation algorithms for multicarrier communication systems," IEEE Trans. Commun., vol. 48, pp. 23-27, Jan. 2002.

[8] P. S. Chow and J. M. Cioffi, "Bandwidth optimization for high speed data transmission over channels with severe intersymbol interference," in proc. IEEE Globecom'92, pp. 59-63, 1992.

[9] P. S. Chow, "Bandwidth optimized digital transmission techniques for spectrally shaped channels with impulse noise," Ph. D. Thesis, Stanford University, 1993.

[10] A. Leke and J. M. Cioffi, "A maximum rate loading algorithm for discrete multitone modulation systems," in proc. IEEE Globecom'97, pp. 1514-1518, 1997.

[11] T. M. Cover and J. A. Thomas, Elements of Information Theory, New York: John Wiley \& Sons, 1991.

[12] A. J. Goldsmith and S. -G. Chua, "Variable-rate variable-power MQAM for fading channels," IEEE Trans. Commun., vol. 45, pp. 1218-1230, Oct. 1997. 